\title{
PCA plus LDA on Wavelet Co-occurrence Histogram Features for Texture Classification and its Applications
}

\author{
Shivashankar S. \\ Dept. of Computer Science \\ Karnatak Science College, Dharwad, \\ Karnataka, India
}

\author{
Hiremath P.S. \\ Dept. of Computer Science, \\ Gulbarga University, Gulbarga, \\ Karnataka, India
}

\begin{abstract}
In this paper, we propose a combined approach, namely, PCA plus LDA on Wavelet Co-occurrence Histogram Features (WCHF) for texture classification. The texture features are extracted using the Wavelet Co-occurrence Histogram (WCH) from wavelet decomposed images, which capture the information about relationships between each high frequency subband and that in low frequency subband of the wavelet transformed image at the corresponding level. The correlation between the subbands at the same resolution exhibits a strong relationship, indicating that this information is significant for characterizing a texture. Thus WCH features thus extracted form a feature vector of dimension 384 for gray scale image which is very high. A combination of Principal Component Analysis (PCA) and Linear Discriminant Analysis (LDA) is applied on $\mathrm{WCH}$ feature vector for dimensionality reduction and enhancement of the class separability respectively. The vectors obtained from the LDA are representative of each image. The classification performance is tested on a set of 32 Brodatz textures. The results are compared with the method proposed in [Hiremath and Shivashankar, 2008]. The effectiveness of proposed method is demonstrated for two different applications, i.e., CBIR and script identification (both printed and handwritten). The classification performance is analyzed using the k-NN classifier. It is evident from the experimental results that the proposed method exhibits superior performance in the reduced feature set.
\end{abstract}

\section{Keywords}

Texture classification; PCA and LDA; Wavelet; CBIR; document image; script identification

\section{INTRODUCTION}

In human vision, the texture analysis plays a significant role in object recognition by the human brain. Thus, in the computer vision, the object recognition algorithms rely on the effectiveness of texture analysis. Textures provide important characteristics for surface and object identification from aerial or satellite photographs, biomedical images and many other types of images. The texture classification is fundamental to many applications such as automated visual inspection, biomedical image processing, content based image retrieval, script identification and remote sensing. Much research work has been done on the texture classification and segmentation for the last four decades. Despite these efforts, the texture classification is still considered an interesting but difficult problem in image processing. Suppose there is a finite number of texture classes $C_{i}, i=1,2,3, \ldots, n$, and a number of training samples of each class are available. Based on the information extracted from the training samples, a decision rule is designed which classifies a given sample of unknown class into one of the $\mathrm{n}$ classes.

To design an effective algorithm for the texture classification, it is essential to find a set of texture features with good discriminating power. More recently, a number of new algorithms for extracting features from the coefficients of a wavelet transform have been proposed in the literature. In each of these feature extraction techniques, the filter coefficients for each subband are analyzed separately. Moreover, the correlation between the bands of the same and that at the different resolution levels is ignored, even though it is well-known that strong relationships between the neighbour bands exist. Portilla and Simoncelli have shown that without knowledge of these relations, accurate reconstruction of the texture is not possible, indicating that this information is significant for characterizing a texture [14]. However, Hiremath and Shivashankar proposed a texture feature extraction method based on the co-occurrence histograms of wavelet decomposed images, which capture the information about relationships between each high frequency subband and that in low frequency subband of the transformed image at the corresponding level. The features extracted form a feature vector of dimensionality 384 for the gray scale image [13]. Despite having excellent discriminative power, the feature set suffers from high dimensionality.

This high dimensionality of the feature vectors creates problems in constructing efficient data structures for classification. One of the problems with high-dimensional feature sets is that, in many cases, not all the measured features are important. For this reason, there is considerable interest in reducing the dimensionality of the descriptors while preserving the original topology of the high dimensional space. An ideal dimensionality reduction technique has the capability of efficiently reducing the data into a lowerdimensional model, while preserving the properties of the original data. The traditional and current state-of-the-art dimension reduction methods are published in the statistics, signal processing and machine learning literature.

In the last decade, Fisher linear discriminant analysis(FLDA) has been demonstrated to be a successful discriminant analysis algorithm in face recognition $[3,4,5,6]$. It performs dimensionality reduction by trying to find a mapping from originally high-dimensional space to a low-dimensional space in which the most discriminant features are preserved. As LDA has been broadly applied and well studied in recent years, a series of LDA algorithms have been developed, the most famous method of which is Fisherface [6, 5]. The PCA plus LDA is found to be an effective framework for linear discriminant analysis(LDA) in high dimensional and singular case[12]. 
Motivated by the success and power of the twophase framework (PCA plus LDA) in pattern classification tasks, the objective of the present paper is to propose PCA plus LDA on Wavelet Co-occurrence Histogram Features (WCHF) for texture classification. The classification performance of the proposed approach is tested on a set of 32 Brodatz textures using k-NN classifier[1]. The results are compared with the method proposed in [13]. It is evident from the experimental results that the proposed method exhibits superior performance in the reduced feature set. The proposed method is applied on two different applications, namely, CBIR and script identification. First, in case of CBIR, the retrieval efficiency is considerably increased when compared to that with the original feature set. Second, in case of document script identification, the proposed scheme has been tested on eight Indian language scripts including English and the results are encouraging. The rest of the paper is organized as follows: In the Section 2, the proposed system is discussed in detail. In Section 3, the results of the classification experiments done on the Brodatz texture images using the proposed are analyzed. The applications to CBIR and script identification problems are discussed in the Section 4. Finally Section 5 concludes the discussion.

\section{PROPOSED METHOD}

The Fig. 1 represents the architecture of the proposed system, which consists of two modules, namely, training module and classification modules, which are described below.

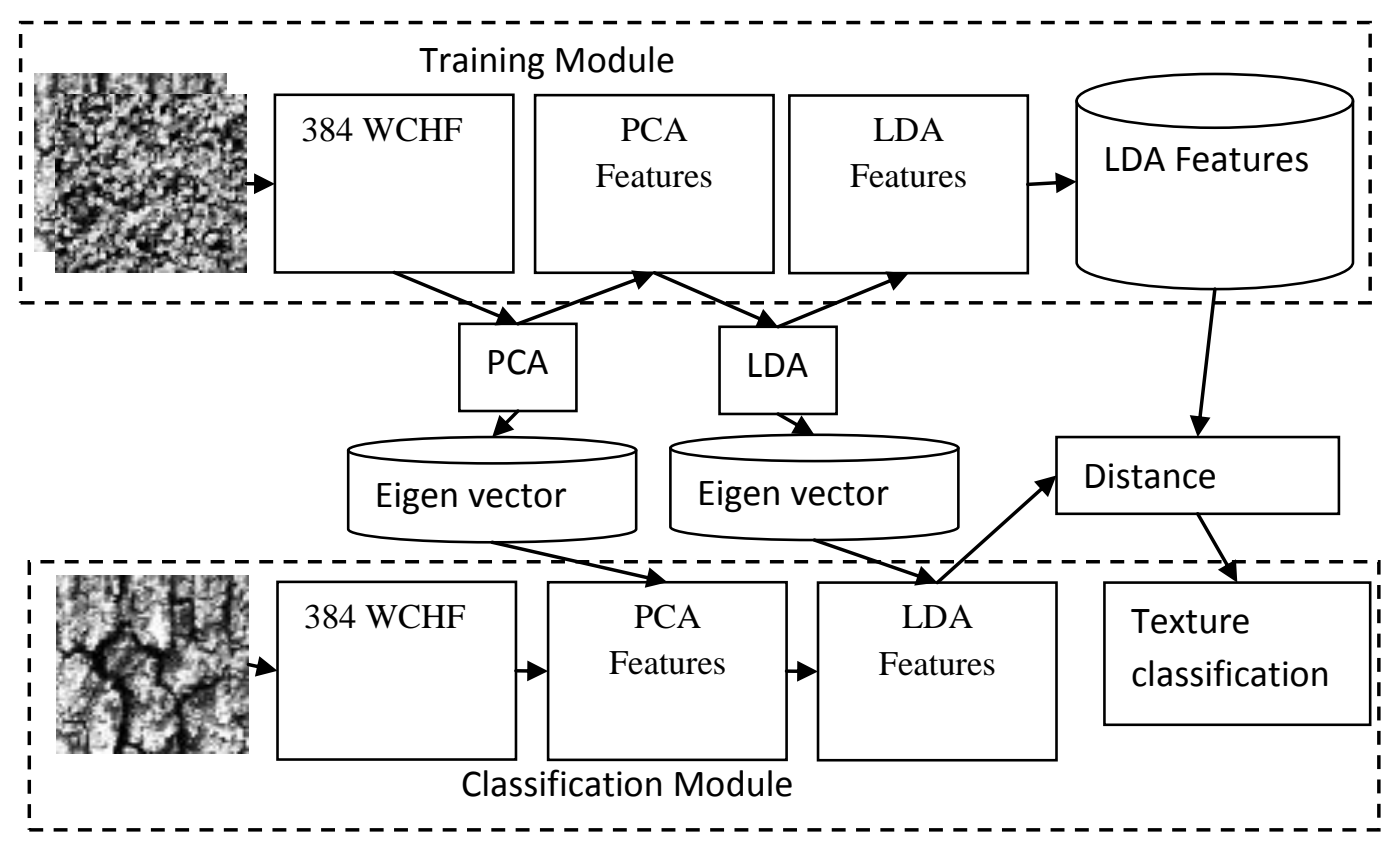

Fig.1 Architecture of the proposed system

\subsection{Texture Training Module}

Each texture image is subdivided into 16 equal sized blocks out of which 8 randomly chosen blocks are used as the training samples and the remaining blocks are used as the test samples for that texture class.

\subsubsection{Wavelet feature}

We extract WCHF from each texture sample [Hiremath and Shivashankar, 2008]. The $\mathrm{i}^{\text {th }}$ texture sample is represented by a $\mathrm{t}$ dimensional vector $x_{i}$ (where $\left.\mathrm{t}=384\right), \mathrm{i}=1,2, \ldots, 8$. These features are extracted using Wavelet Co-occurrence Histogram (WCH) from wavelet decomposed images, which capture the information about relationships between each high frequency subband and that in low frequency subband of the transformed image at the corresponding level. The correlation between the subbands at the same resolution exhibits a strong relationship, indicating that this information is significant for characterizing a texture. The vectors obtained in this manner from all 8 training samples will be denoted by the feature vector $X=\left\{x_{1}, x_{2} \ldots, x_{8}\right\}$. Further these features are used for dimensionality reduction.

\subsubsection{The PCA subspace}

Given a set of data, the PCA finds the linear lowerdimensional representation of the data such that the variance of the reconstructed data is preserved $[9.10,11]$. So, the main idea behind using PCA in our approach is to reduce the dimensionality of the WCHF.

Given a t dimensional vector representation of each sample, the PCA is used to find a subspace whose basis vectors correspond to the maximum-variance directions in the original space. Let $\mathrm{W}$ represent the linear transformation that maps the original t-dimensional space onto a f-dimensional feature subspace where normally $f \ll t$. The new feature vectors $y_{i} \in \mathfrak{R}^{f}$ are defined by $y_{i}=W^{T} x_{i}, i=1, \ldots, N$. The columns of $\mathrm{W}$ are the eigenvectors $e_{i}$ obtained by solving the eigenstructure decomposition $\lambda_{i} e_{i}=Q e_{i}$, where $\mathrm{Q}=X X^{T}$ is the covariance matrix and $\lambda_{i}$ the eigenvalue associated with the eigenvector $e_{i}$. Before obtaining the eigenvectors of $\mathrm{Q}$, the eigen vectors are normalized such that $\left\|x_{i}\right\|=1$ in order to make the system invariant to the intensity of the illumination source. The eigenvectors are stored in the database and are called as PCA eigenvectors. A PCA feature vector is obtained with reduced dimension of 40 features for each image. 


\subsubsection{The LDA subspace}

The LDA searches for those vectors in the underlying space that best discriminate among classes (rather than those that best describe the data). Thus, given a number of independent features relative to which the data is described, LDA creates a linear combination of these which yields the largest mean differences between the desired classes. Mathematically speaking, for all the samples of all classes, we define two measures; namely, within-class scatter matrix and betweenclass scatter matrix.

The within-class scatter matrix $S_{w}$, is defined by

$$
S_{w}=\sum_{j=1}^{C} \sum_{i=1}^{N_{j}}\left(y_{i}^{j}-\mu_{j}\right)\left(y_{i}^{j}-\mu_{j}\right)^{T},
$$

where $y_{i}^{j}$ is the $i^{\text {th }}$ sample of class $\mathrm{j}, \mu_{j}$ is the mean of class $\mathrm{j}$, $\mathrm{C}$ is the number of classes, and $N_{j}$ the number of samples in class $\mathrm{j}$.

The between-class scatter matrix $S_{b}$ is defined by

$$
S_{b}=\sum_{j=1}^{C}\left(\mu_{j-1} \mu\right)\left(\mu_{j}-\mu\right)^{T},
$$

where $\mu$ represents the mean of all classes.

The goal is to maximize the between-class measure while minimizing the within-class measure, which is achieved by maximizing the ratio $\frac{\operatorname{det}\left|S_{b}\right|}{\operatorname{det}\left|S_{w}\right|}$. The advantage of using this ratio is that it has been proven [8], if $S_{w}$ is a nonsingular matrix then this ratio is maximized when the column vectors of the projection matrix, W, are the eigenvectors of $S_{w}{ }^{-1} S_{b}$. It should be noted that: (i) There are at most $\mathrm{C}-1$ nonzero generalized eigenvectors and, so, an upper bound on $\mathrm{f}$ is $\mathrm{C}$ - 1,(ii) We require at least $\mathrm{f}+\mathrm{C}$ samples to guarantee that $S_{w}$ does not become singular (which is almost impossible in any realistic application). To solve this, [7] and [10] have proposed to use PCA subspace as an intermediate space. Thus, the original t-dimensional space is projected onto an intermediate f-dimensional space using PCA and then onto a final g-dimensional space using LDA. These LDA features for each image are stored in the database, which is further used for texture classification.

\subsection{Texture Classification Module}

In the classification phase, the 384 WCHF are extracted from the test sample as described in the Section 2.1.1. These features are projected on PCA eigen vectors to obtain PCA feature subspace of dimensionality 40 as explained in the Section 2.1.2. Further, these features are projected on LDA eigen vectors, which generate LDA feature subspace as explained in the Section 2.1.3. These LDA features are compared with those stored in the feature database by using the Euclidian distance.

$$
D(M)=\sqrt{\sum_{j=1}^{N}\left(f_{j}[X]-f_{j}[M]\right)^{2}}
$$

where, $\mathrm{N}$ is the number of features, $f_{j}[X]$ represents $j^{\text {th }}$ feature of the test sample $\mathrm{X}$, while $f_{j}[M]$ represents the $j^{\text {th }}$ feature of the $M^{\text {th }}$ texture class in the feature database. Then, the test texture $\mathrm{X}$ is classified using the k-Nearest Neighbor classifier [15]. In the $\mathrm{k}-\mathrm{NN}$ classifier, a test sample is classified by a majority vote of its neighbors, with the test sample being assigned the class most common among its $\mathrm{k}$ nearest neighbors, where $\mathrm{k}$ is a positive integer, it is helpful to choose $\mathrm{k}$ to be an odd number as this avoids difficulties with tied votes.
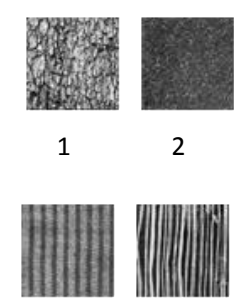

9
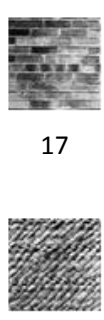

25

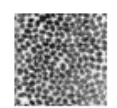

3

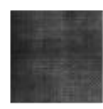

11

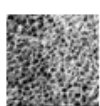

18

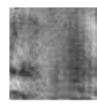

26

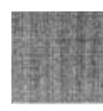

19

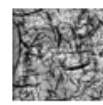

27

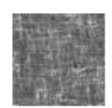

4

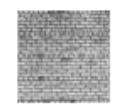

12

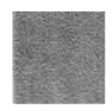

20

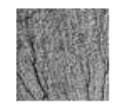

28

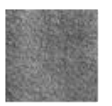

5

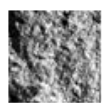

13

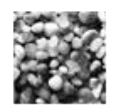

21

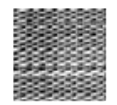

29
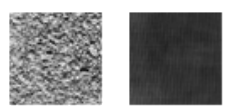

6

7

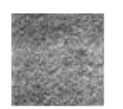

14

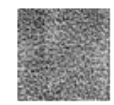

22

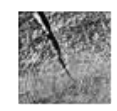

30

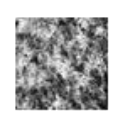

15

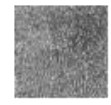

23

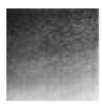

31

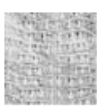

8

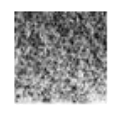

16

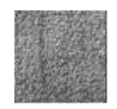

24

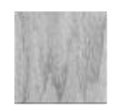

32

Fig. 2 Texture images from Brodatz album. The image Nos 1 to 32 correspond to the texture Sl.No. 1 to 32 in Table 1, respectively. 


\section{EXPERIMENTAL RESULTS}

\subsection{Experimental data}

In order to assess the discrimination capability of a wavelet based feature set, we have performed experiments tests using the same data presented in [13], where we define 32 test categories from the selected images of Brodatz (1966) collection as shown in the Fig. 2. For the 32-category problem, the texture samples are obtained from a 256X256 image with 256 gray levels. Each image is subdivided into 16 blocks of 64 X64 pixels and each block is transformed into a new block by $90^{\circ}$ rotation. This produced 1024 blocks. Half of the data set is selected by randomly choosing 8 blocks and the corresponding transformed blocks, which define the texture classes. The remaining blocks are used to evaluate the classification process.

\subsection{Classification results}

The Table 1 represents classification performance for different approaches using $\mathrm{k}-\mathrm{NN}$ classifier with $\mathrm{k}=3$. The WCHF(384) denotes the classification accuracy with wavelet cooccurrence histogram features of dimensionality 384, the PCA(10) specifies the classification accuracy after applying the PCA on WCHF and considering only first 10 eigen vectors after projecting the features on eigen vectors, whereas PCA_LDA(3), PCA_LDA(2), PCA_LDA(1) denote the classification accuracies after applying LDA on PCA and considering only 3,2,1 eigen values, respectively. It is evident from the results that the proposed method exhibits superior performance in the reduced feature subspace. The classification efficacy is $100 \%$ for proposed method based on only 3 features whereas it is $96.84 \%$ for the original feature set which is based on wavelet co-occurrence histogram having 384 features.

Table-1 - Average classification accuracies (\%) over 10 experiments for 32 texture category

\begin{tabular}{|c|c|c|c|c|c|c|}
\hline \multirow{2}{*}{ Sl. No. } & \multirow{2}{*}{ Texture } & \multicolumn{5}{|c|}{ Correct classification (in \%) } \\
\hline & & WCHF(384) & PCA(10) & PCA_LDA(3) & PCA_LDA(2) & PCA_LDA(1) \\
\hline 1 & Bark & 98.75 & 30 & 100 & 100 & 97.5 \\
\hline 2 & Beachsand & 97.5 & 47.5 & 100 & 100 & 97.5 \\
\hline 3 & Beans & 100 & 71.25 & 100 & 100 & 92.5 \\
\hline 4 & Burlap & 100 & 30 & 100 & 100 & 88.75 \\
\hline 5 & D10 & 98.75 & 17.5 & 100 & 100 & 93.75 \\
\hline 6 & D11 & 100 & 51.25 & 100 & 100 & 100 \\
\hline 7 & D4 & 100 & 56.25 & 100 & 100 & 85 \\
\hline 8 & D5 & 100 & 32.5 & 100 & 100 & 91.25 \\
\hline 9 & D51 & 100 & 27.5 & 100 & 100 & 93.75 \\
\hline 10 & D52 & 100 & 27.5 & 100 & 100 & 95 \\
\hline 11 & D6 & 100 & 42.5 & 100 & 100 & 80 \\
\hline 12 & D95 & 100 & 5 & 100 & 98.75 & 76.25 \\
\hline 13 & Fieldstone & 100 & 40 & 100 & 100 & 90 \\
\hline 14 & Grass & 92.5 & 50 & 100 & 100 & 91.25 \\
\hline 15 & Ice & 96.25 & 40 & 100 & 100 & 93.75 \\
\hline 16 & Image09 & 100 & 35 & 100 & 100 & 83.75 \\
\hline 17 & Image 15 & 83.75 & 2.5 & 100 & 100 & 86.25 \\
\hline 18 & Image17 & 100 & 40 & 100 & 100 & 91.25 \\
\hline 19 & Image19 & 100 & 40 & 100 & 100 & 98.75 \\
\hline 20 & Paper & 100 & 51.25 & 100 & 100 & 91.25 \\
\hline 21 & Peb54 & 95 & 11.25 & 100 & 100 & 88.75 \\
\hline 22 & Pigskin & 96.25 & 33.75 & 100 & 100 & 93.75 \\
\hline 23 & Pressedcl & 100 & 5 & 100 & 100 & 97.5 \\
\hline 24 & Raffia & 100 & 63.75 & 100 & 97.5 & 87.5 \\
\hline 25 & Raffia2 & 100 & 33.75 & 100 & 100 & 90 \\
\hline 26 & Reptile & 98.75 & 32.5 & 100 & 100 & 91.25 \\
\hline 27 & Ricepaper & 73.75 & 12.5 & 100 & 100 & 97.5 \\
\hline 28 & Seafan & 100 & 32.5 & 100 & 100 & 90 \\
\hline 29 & Straw2 & 100 & 45 & 100 & 100 & 98.75 \\
\hline 30 & Tree & 81.25 & 2.5 & 100 & 100 & 90 \\
\hline 31 & Water & 97.5 & 16.25 & 100 & 100 & 95 \\
\hline 32 & Woodgrain & 88.75 & 7.5 & 100 & 98.75 & 92.5 \\
\hline Mean & ccess rate & 96.84 & 32.30 & 100 & 99.84 & 91.56 \\
\hline
\end{tabular}




\section{APPLICATIONS}

The efficacy of the proposed method for texture classification is demonstrated by considering two applications, namely, content based image retrieval (CBIR) and document image script identification.

\subsection{Application to CBIR}

With an ever-increasing use and demand for digital images in the areas of medicine, engineering, sciences, digital photography, and advertisement. The importance of contentbased image retrieval (CBIR) is on the rise and is considered as a subject of intense research [18]. The CBIR technique focuses on searching for images in database which are similar to the query image according to the image features related to content. This technique is based on automatic extraction of image features, and then image retrieval automatically by comparing the features of query image (such as color, shape, texture, etc.) with the corresponding features in image feature library, and finally presents the best matching images and its corresponding information.

The proposed system is implemented on WANG database[17], which is a subset of Corel images consisting of 1000 images of natural scenes divided into 10 labeled categories of 100 images each. These images were arranged in 10 semantic groups: tribesmen, elephants, horses, flowers, foods, greek architecture, buses, Dinosaurs, mountains, and beaches.

In these experiments, the features are extracted by considering whole image ( i.e., non-segmented approach). The experimental results of the proposed method are compared with WCHF extracted on each of the Red, Green and Blue bands (1152 features), PCA (40 features) and PCA+LDA (39 features). The Fig. 3 shows a precesion recall graph with the averaging done over 10 query images one image randomly choosen from each category. It is evident that the result of the proposed method outperforms the other two methods in the reduced feature set (i.e., retrieval efficiency is $87 \%$ for proposed method, $66 \%$ for PCA and $35 \%$ for original set based on wavelet features). The precesion recall graph for few queries are shown in the Fig. 4-7. The Fig. 8 shows the sample query images and top 10 retrievals for each query obtained by the proposed method. The first image is the query image, followed by the corresponding retrieved images. We are able to retrieve all the top 10 images for the categories, namely, buses, Dinosaurs, flowers, horses and mountains. For the greek architecture, elephants and food categories the top 9 images are of the same category, the top 8 images are from the tribesman category, whereas a poor results of only 6 images are retrieved for beaches category.

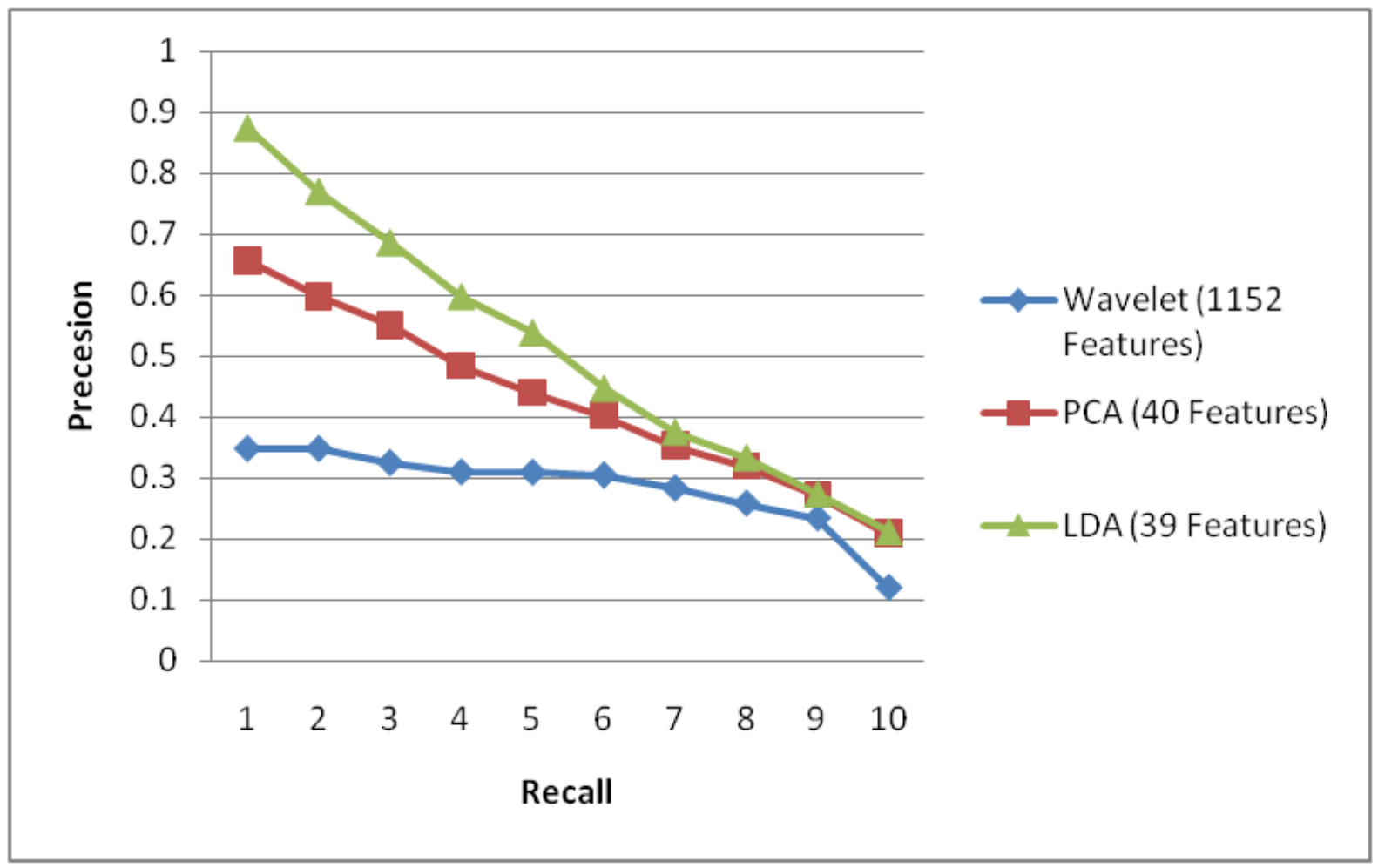

Fig 3: Average Precesion recall graph of 10 images, one image choosen from each category 


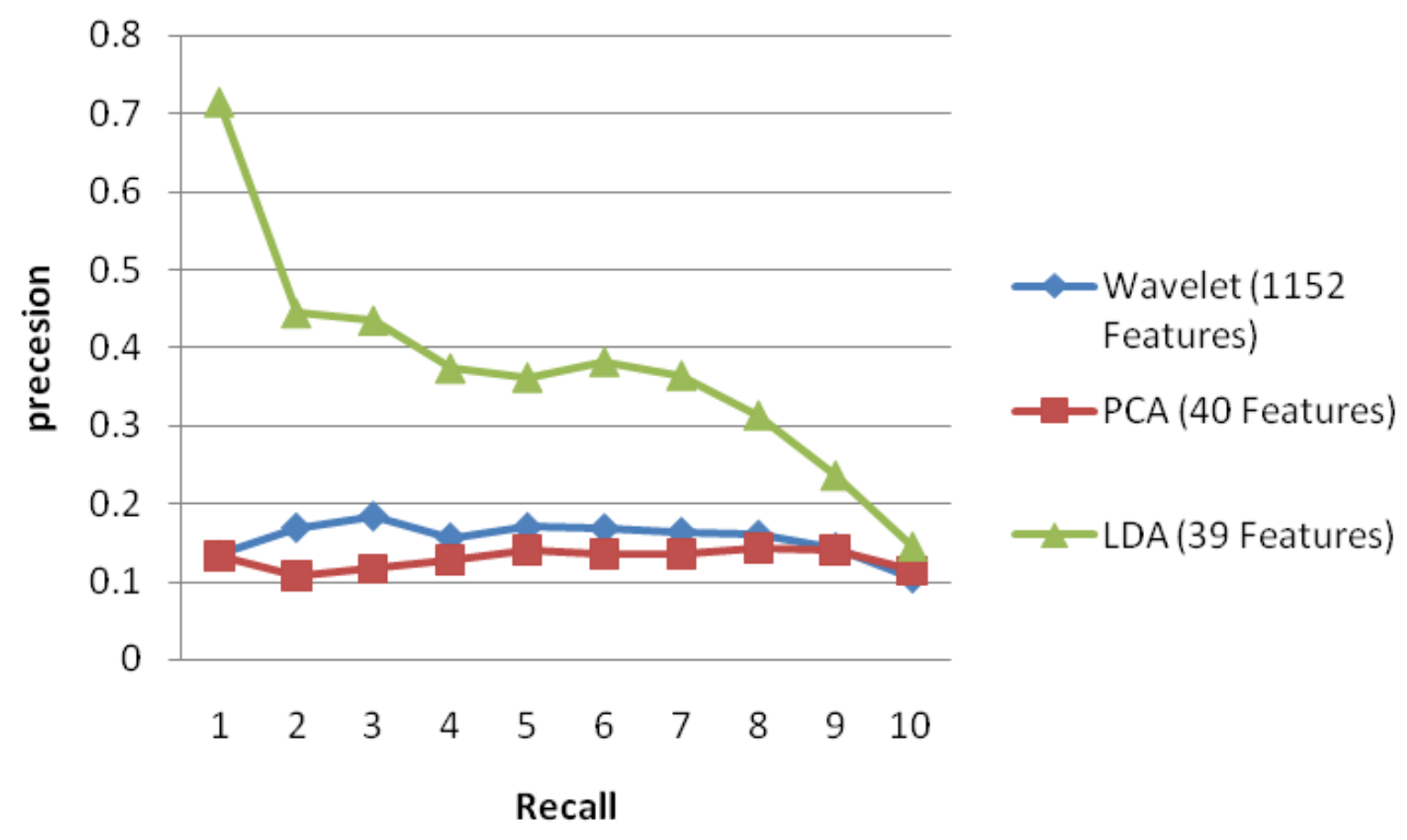

Fig 4: Precesion recall graph for tribesmen (43)

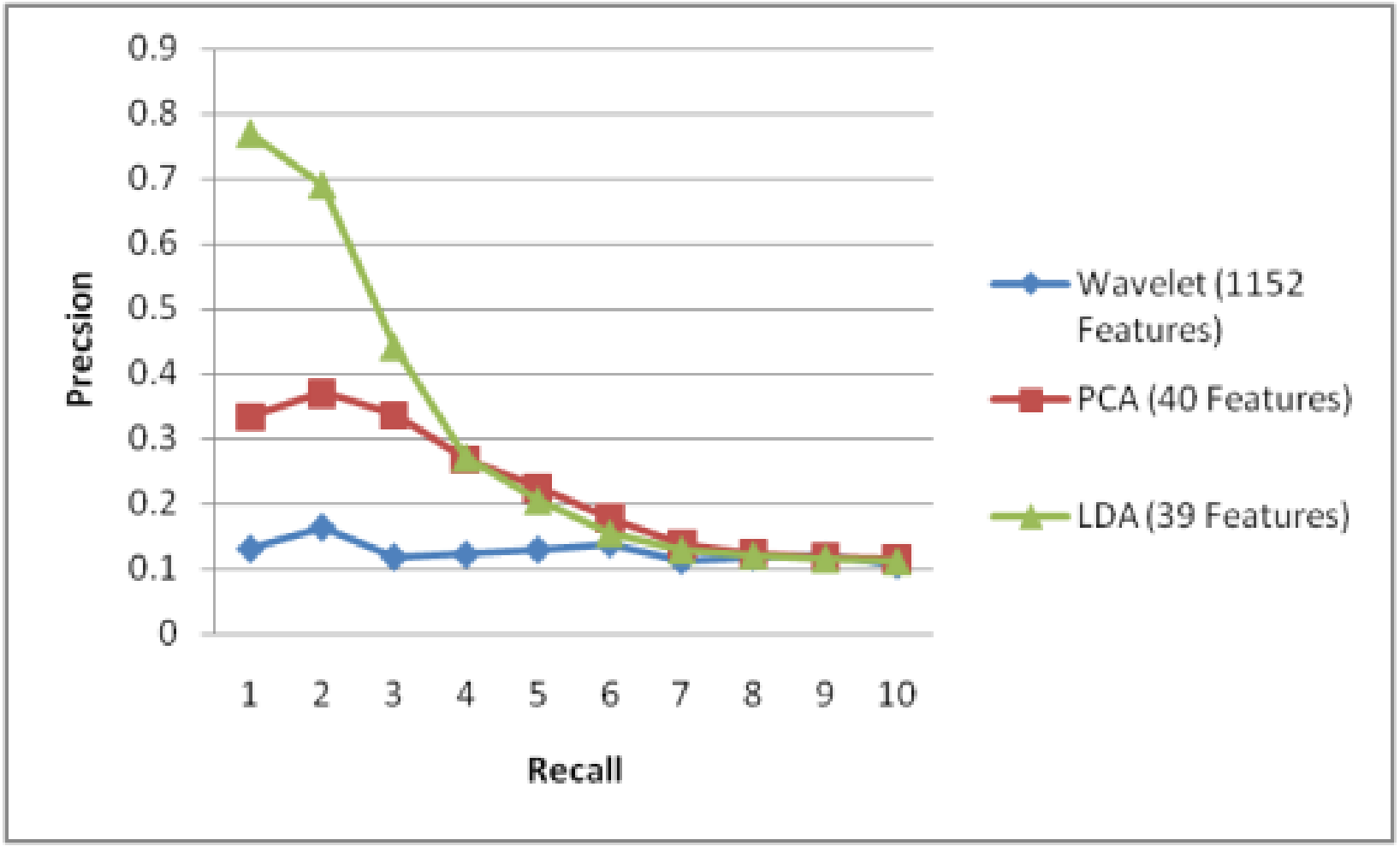

Fig 5: Precesion recall graph for Monuments(204) 


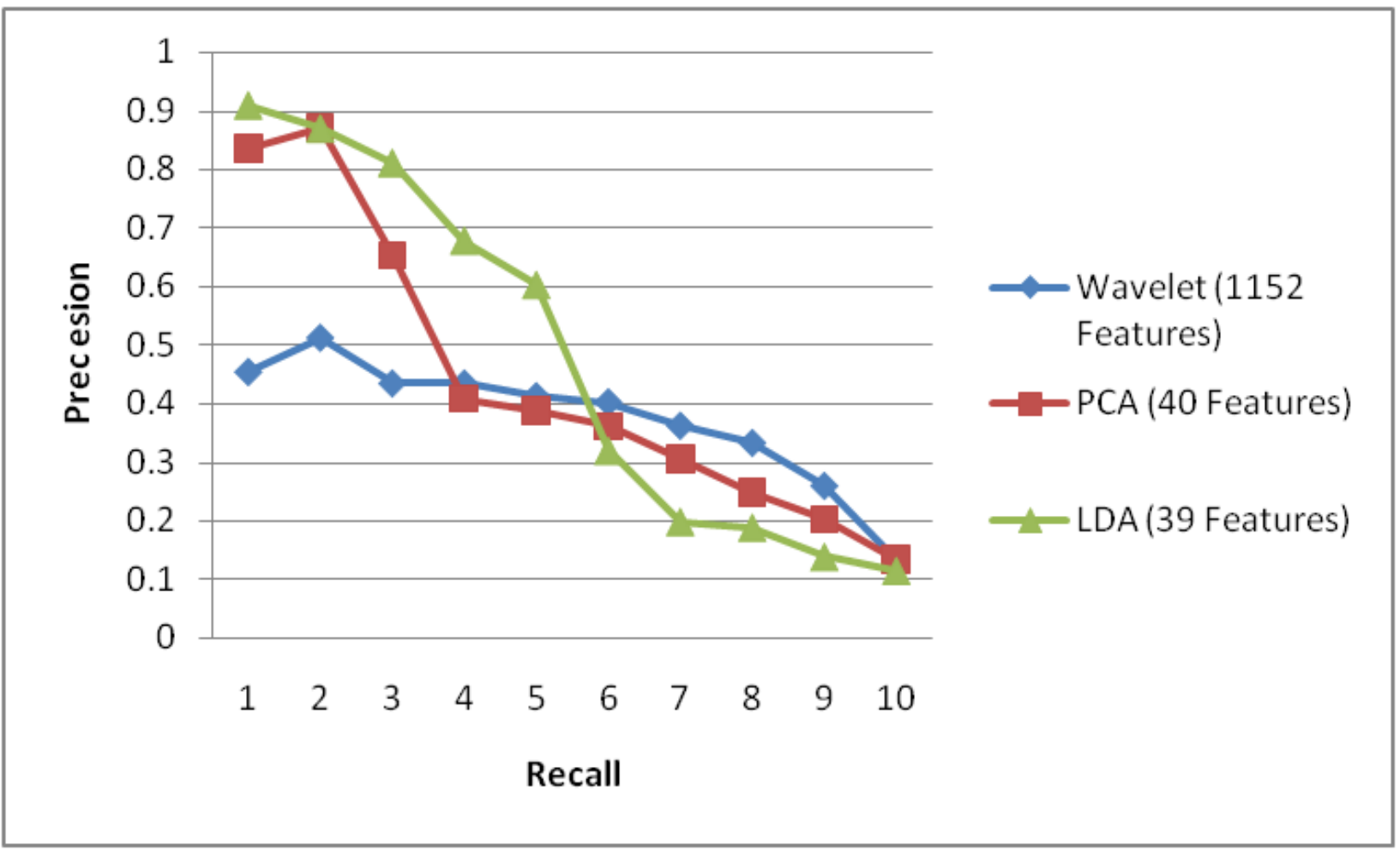

Fig 6: Precesion recall for graph Elephant (510)

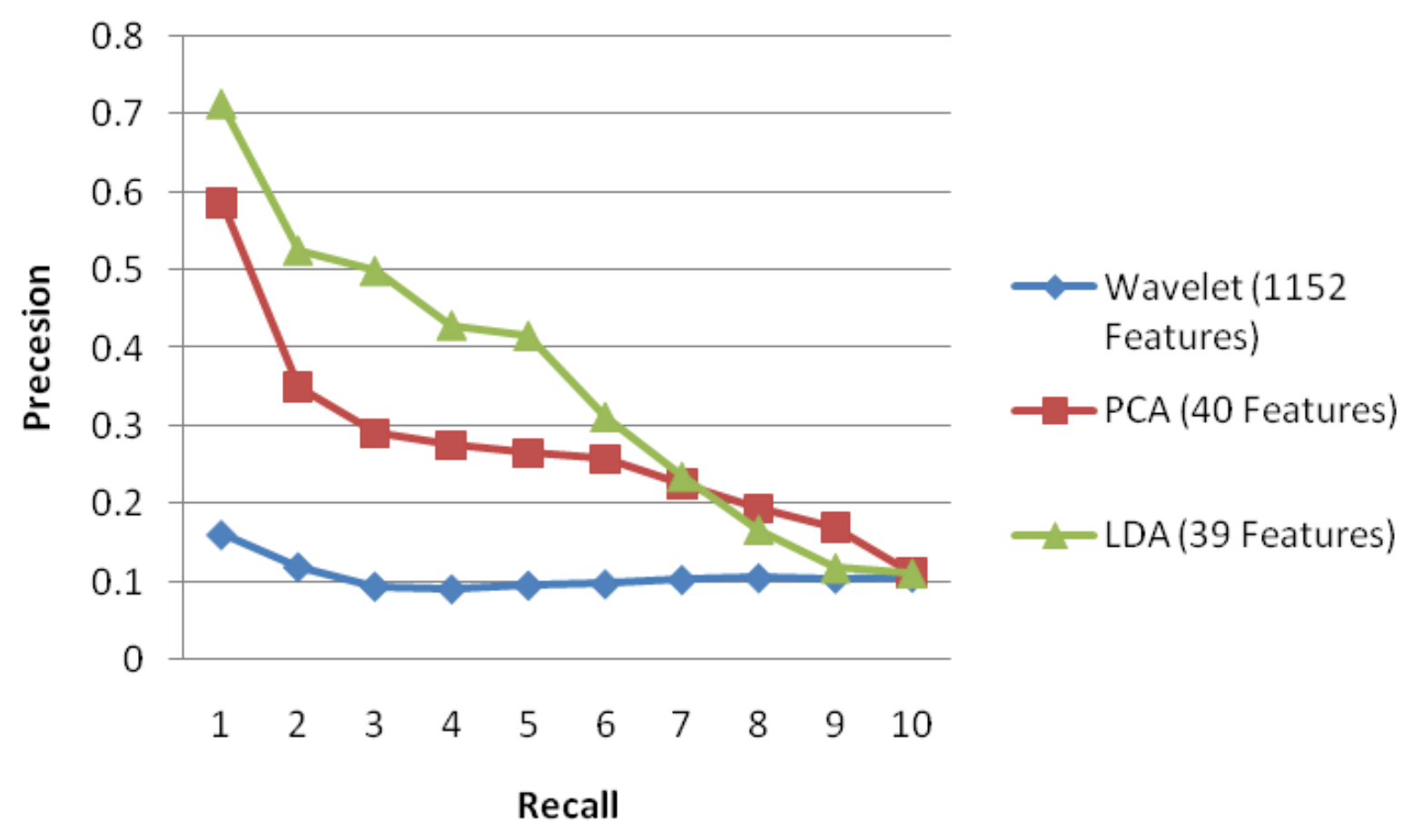

Fig 7: Precesion recall graph for Food(955) 


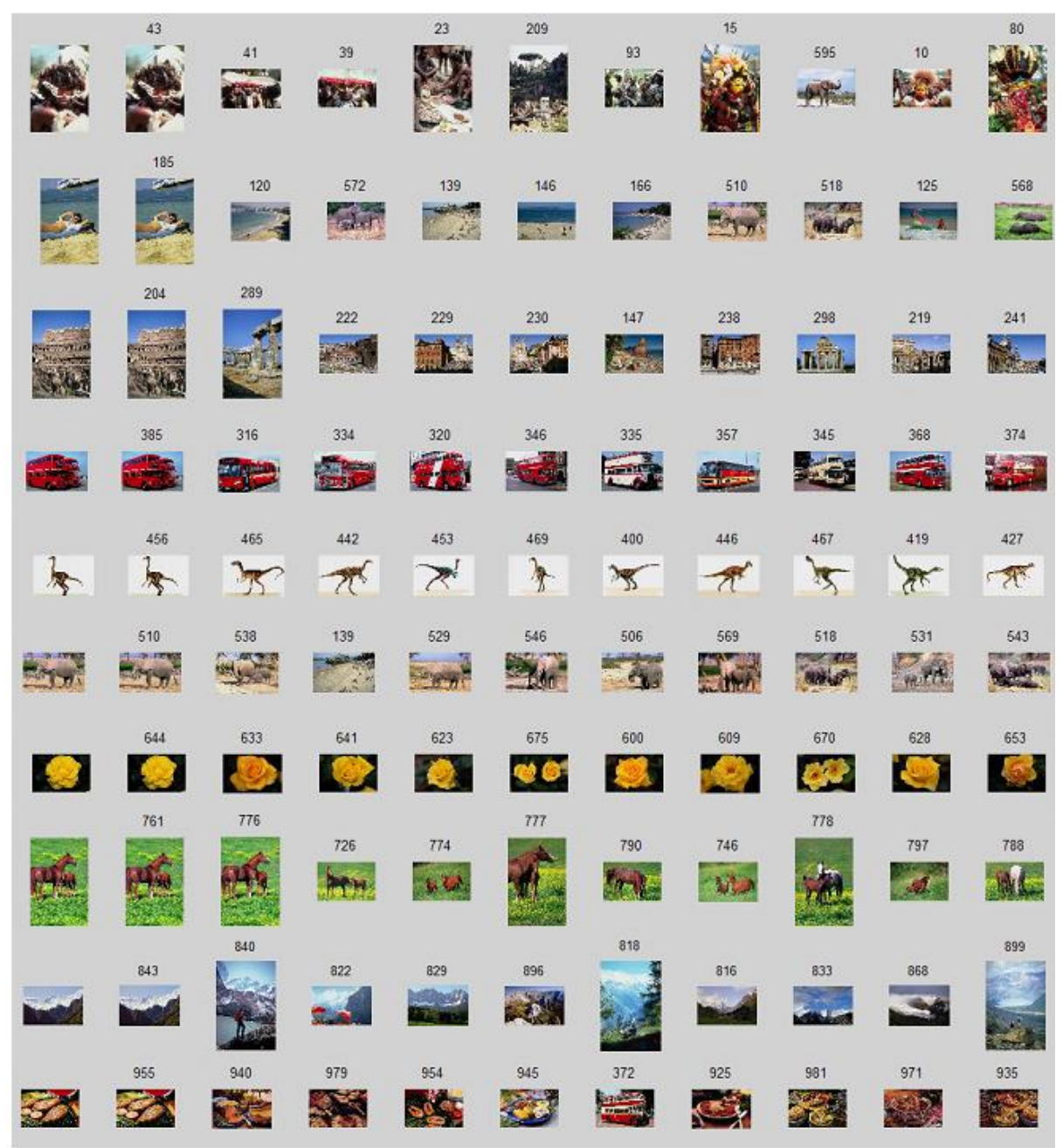

Fig. 8 Sample query image followed by top ten retrievals in ten examples.

\subsection{Application to Script Identification}

Although a great number of optical character reader (OCR) techniques have been developed over the years, almost all the existing works on OCR make an important implicit assumption that the script and/or language of the document to be processed is known a priori. In practice, this implies that human intervention in identifying the script/language of each document is essential, which severely restricts its utility. For minimal human involvement, there should be an automatic mechanism where by the language of the input document is first identified and the appropriate OCR module is then selected. Since each script often has a distinctive visual appearance, a block of text in a script document (regardless of content) may be considered as a texture. Therefore, the problem of script identification may be tackled by means of the texture classification $[2,16]$. This observation motivates us to utilize the proposed texture classification algorithm for the script identification. A texture based approach does not require connected component analysis and hence may be called a global approach. The proposed texture features are tested on both printed and handwritten document images for script identification separately. English and seven Indian scripts (namely, Kannada, Tamil, Urdu, Telugu, Bengali, Hindi, and Malayalam) are used in the experiments. The script documents are digitized at $150 \mathrm{dpi}$. The experiments were carried out for 1600 images with different orientations. The sample images of the printed and handwritten scripts used in experimentation are shown in Fig. 9 and Fig.10, respectively. As in the texture classification problem, half the numbers of samples are used for training and the remaining half for testing. The image size of the script samples used for the experiments is $128 \mathrm{X} 128$ pixels. These images are binarized using global threshold. The results of these experiments are given in the Tables 2 and 3. The average classification accuracy is $100 \%$ for features extracted by the proposed PCA+LDA on WCHF using only 2 features, which is higher than that of the method using only WCHF with 384 features and PCA with 3 features. These results demonstrate the efficacy of the proposed combined approach namely, PCA plus LDA on wavelet based feature set (WCHF) in the script identification. 

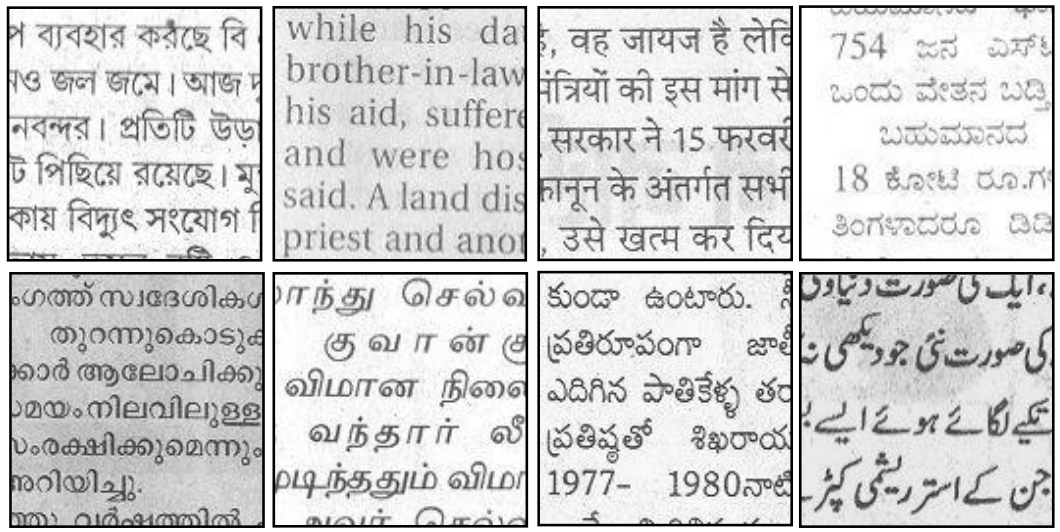

Fig. 9. Sample image of the Printed Scripts used for identification problem

(Left to right row 1: Bengali, English, Hindi, Kannada, row 2: Malayalam, Tamil, Telugu, Urdu)
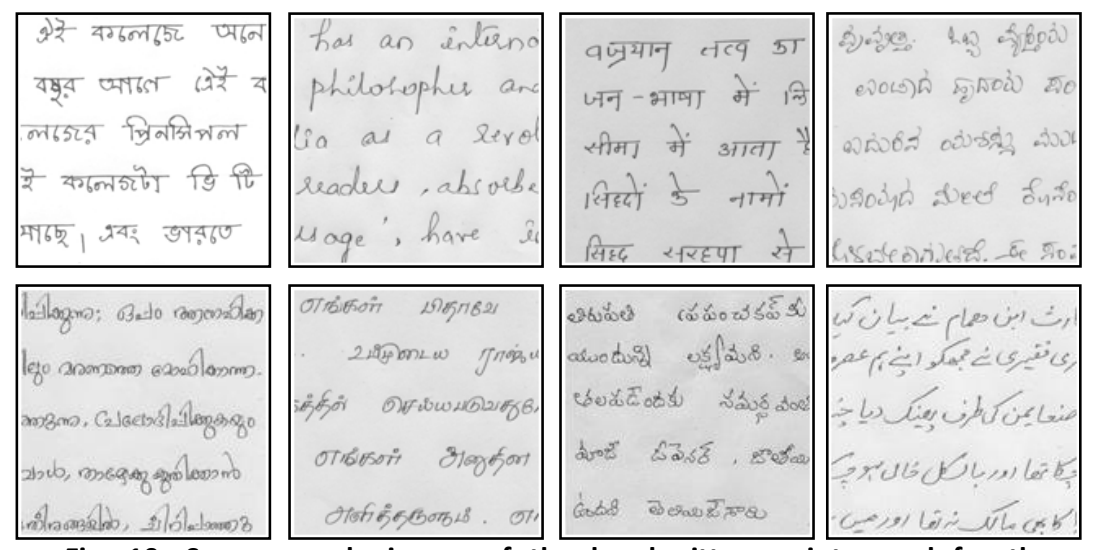

Fig. 10. Some sample image of the handwritten scripts used for the identification problem (Left to right row 1: Bengali, English, Hindi, Kannada, row 2: Malayalam, Tamil, Telugu, Urdu)

Table 2. Average classification accuracies (\%) for different printed scripts

\begin{tabular}{|c|c|c|c|c|}
\hline & Wavelet & PCA(3) & PCA_LDA(1) & PCA_LDA(2) \\
\hline Bengali & 86.4 & 99.1 & 98.8 & 100 \\
\hline English & 90.4 & 99.8 & 99.2 & 100 \\
\hline Hindi & 96.1 & 99.9 & 100 & 100 \\
\hline Kannada & 88.8 & 99.3 & 96.1 & 100 \\
\hline Malayalam & 93.3 & 99.6 & 96.9 & 100 \\
\hline Tamil & 85 & 98.7 & 99.5 & 100 \\
\hline Telgu & 86.9 & 98.1 & 97 & 100 \\
\hline Urdu & 99.7 & 100 & 100 & 100 \\
\hline Average & $\mathbf{9 0 . 8 2 5}$ & $\mathbf{9 9 . 3 1}$ & $\mathbf{9 8 . 4 3 8}$ & $\mathbf{1 0 0}$ \\
\hline
\end{tabular}


Table 3. Average classification accuracies (\%) for different hand written scripts

\begin{tabular}{|c|c|c|c|c|}
\hline & Wavelet & PCA(3) & PCA_LDA(1) & PCA_LDA(2) \\
\hline Kannada & 99.6 & 100 & 97.7 & 100 \\
\hline English & 100 & 100 & 100 & 100 \\
\hline Tamil & 83.6 & 92.5 & 99.9 & 100 \\
\hline Urdu & 100 & 100 & 100 & 100 \\
\hline Telugu & 96.8 & 97.5 & 100 & 100 \\
\hline Bengali & 100 & 100 & 100 & 100 \\
\hline Hindi & 100 & 100 & 97 & 100 \\
\hline Malayalam & 100 & 100 & 100 & 100 \\
\hline Average & $\mathbf{9 7 . 5}$ & $\mathbf{9 8 . 7 5}$ & $\mathbf{9 9 . 3 2 5}$ & $\mathbf{1 0 0}$ \\
\hline
\end{tabular}

\section{CONCLUSION}

In the computer vision, the object recognition algorithms rely on the effectiveness of texture analysis. In this paper, a combined approach, namely, proposed PCA plus LDA on Wavelet Co-occurrence histogram features for texture classification is proposed. Wavelet co-occurrence histogram features capture the information about relationships between each high frequency subbands and that in low frequency subband of the transformed image at the corresponding level. From the experimental results, we observe that:

1. The texture classification performance is improved upto $100 \%$ with only 3 features.

2. In case of CBIR, the retrieval efficiency is $87 \%$ for proposed method with 39 features as compared to $66 \%$ for PCA with 40 features and $35 \%$ for original 1152 WCHF.

3 . In case of both printed and handwritten document images, the script identification is $100 \%$ for proposed method with only 2 features.

It is evident from experimental results that the proposed method is effective in terms of both dimension reduction and the classification accuracy in comparison with PCA and WCHF.

\section{ACKNOWLEDGEMENTS}

Authors are indebted to the reviewers for their helpful comments.

\section{REFERENCES}

[1]. Brodatz, P., 1966. Textures: A Photographic Album for Artists and Designers. Dover, New York.

[2]. Busch, A., Boles, W.W., Sridharan, S., 2005. Texture for script identification. IEEE Trans. Pattern Anal. Machine Intell. 27 (11), 1720-1732.

[3]. C. Chiu, H. Lin, S. Yang., 2003. A Fuzzy Logic CBIR System. The IEEE International Conference on Fuzzy Systems. pp. 1171- 1176.

[4]. C.M. Bishop.,1995. Neural Networks for Pattern Recognition. Oxford University Press.

[5]. Carlton W. Niblack, Ron Barber, Will Equitz, Myron D. Flickner, Eduardo H. Glasman, Dragutin Petkovic, Peter Yanker, Christos Faloutsos, and Gabriel Taubin, 1993. QBIC project: querying images by content, using color, texture, and shape. Storage and Retrieval for Image and Video Databases, 1908(1):173-187.
[6]. Chang, T., and C.C.J.Kuo. 1993. Texture analysis and classification with tree-structured wavelet transform. IEEE Trans. on Image Processing 2(4):429-441.

[7]. Crandell. 1994. Projects in scientific computation. Springer-Verlag, New York:197- 198,211-212.

[8]. D. J. Hand., 1981. Discrimination and Classification. New York: John Wiley.

[9]. D. Swets, and J. Weng, Oct. 1996. "Discriminant Analysis and Eigenspace Partition Tree for Face and Object Recognition from Views, "In Proc. IEEE International Conference on Automatic Face and Gesture Recognition, pp.192 - 197.

[10].D.L. Swets and J.J. Weng, Aug. 1996. "Using Discriminant Eigenfeatures for Image Retrieval," IEEE Trans. Pattern Analysis and Machine Intelligence, vol. 18, no. 8, pp. 831-836.

[11].F. Li, Q. Dai, W. Xu. , 2006. Improved Similarity-Based Online Feature Selection In Region-Based Image Retrieval. IEEE. ICME. pp. 349-352 .

[12].Jian Yang, Jing-yu Yang, 2003. "Why can LDA be performed in PCA transformed space", Pattern Recognition, 36, 563-566.

[13].P.S. Hiremath, S. Shivashankar, 2008. "Wavelet based co-occurrence histogram features for texture classification with an application to script identification in a document image", Pattern Recognition Letters 29, $1182-1189$.

[14].Portilla, Javier, Simoncelli, E.P., 2000. A parametric texture model based on joint statistics of complex wavelet coefficients. Internat. J. Comput. Vision 40 (1), 49-70.

[15].R.O. Duda, P.E. Hart, D.G. Stork, 2001. Pattern Classification, Wiley, New York.

[16].Tan, T.N., 1998. Rotation invariant texture features and their use in automatic script identification. IEEE Trans. Pattern Anal. Machine Intell. 20 (7), 751-756.

[17].Wang database, http://wang.ist.psu.edu/.

[18].Ying Liua, Dengsheng Zhanga, Guojun Lua,Wei-Ying Mab, 2007. "A survey of content-based image retrieval with high-level semantics", Pattern Recognition 40, 262 -282 . 